\title{
Calendar of Events
}

European Academy of Childhood Disability $26^{\text {th }}$ Annual Meeting of the European Academy of Childhood Disability

\section{July 3-5, 2014}

Vienna, Austria

Website: http://www.eacd2014.org

The American Academy of Cerebral Palsy and Developmental Medicine (AACPDM) $68^{\text {th }}$ Annual Meeting

\section{September 10-13, 2014}

Hilton San Diego Bayfront San Diego, CA

Website: http://www.aacpdm.org/meetings/2014

American Society of Hand Therapists (ASHT)

ASHT's 37th Annual Meeting

\section{September 18-20, 2014}

Queen Elizabeth II Conference Centre

Westminster, London

Website: http://www.cribsafety.jpma.org

American Academy of Pediatrics

AAP National Conference and Exhibition (NCE)

October 11-14, 2014

San Diego, CA

Website:

http://s15.a2zinc.net/clients/AAP/NCE2014/Public/
Congress of Neurological Surgeons Annual Meeting

October 18-22, 2014

Boston, MA

Website: http://w3.cns.org/meetings/2014/index.asp

American Academy of Physical Medicine and Rehabilitation (AAPM \& R)

Annual Assembly and Technical Exhibition

November 13-16, 2014

San Diego Convention Center

San Diego, CA

Website:

http://www.aapmr.org/assembly/Pages/default.aspx

American Physical Therapy Association

Combined Sections Meeting (CSM)

February 4-7, 2015

Indianapolis, Indiana, USA

Website: http://www.apta.org/csm

Arthroscopy Association of North America (AANA)

Metcalf/AANA Arthroscopic Surgery Seminar

\section{March 5-8, 2015}

Snowbird, UT

Website:

http://www.posna.org/meetings/anmeet/anmeet.asp 
Association of Academic Physiatrists

2015 AAP Annual Meeting

March 10-14, 2015

San Antonio, TX

Website:

http://www.physiatry.org/?page=meeting_2015

American Occupational Therapy Association

$95^{\text {th }}$ Annual Conference \& Expo

April 16-19, 2015

Nashville, TN

Website: http://www.aota.org/ConfandEvents.aspx
American Academy of Neurology

66th Annual Meeting

April 18-25, 2015

Washington, DC

Website: http://www.aan.com/go/home

American Urological Association

AUA Annual Meeting

May 15-19, 2015

New Orleans, LA, USA

Website: http://www.aua2015.org 